\title{
High-energy QCD evolution from BRST symmetry
}

\author{
D. Binosi ${ }^{1, \mathrm{a}}$, A. Quadri ${ }^{2,3, \mathrm{~b}}$, D. N. Triantafyllopoulos ${ }^{1, \mathrm{c}}$ \\ ${ }^{1}$ European Centre for Theoretical Studies in Nuclear Physics and Related Areas (ECT*) and Fondazione Bruno Kessler, \\ Strada delle Tabarelle 286, 38123 Villazzano (TN), Italy \\ ${ }^{2}$ Dip. di Fisica, Università degli Studi di Milano, Via Celoria 16, 20133 Milano, Italy \\ ${ }^{3}$ INFN, Sezione di Milano, Via Celoria 16, 20133 Milano, Italy
}

Received: 24 February 2014 / Accepted: 29 May 2014 / Published online: 19 June 2014

(C) The Author(s) 2014. This article is published with open access at Springerlink.com

\begin{abstract}
We show that the (gauge-fixed) classical action of the Color Glass Condensate is invariant under a suitable Becchi-Rouet-Stora-Tyutin symmetry, which holds after the gluon modes are split into their fast, semi-fast, and soft components, according to the longitudinal momenta they carry. This entails the existence of a corresponding SlavnovTaylor identity which in turn strongly constrains the effective field theory arising when integrating out the semi-fast modes. Specifically, we prove that this identity guarantees the gauge invariance of the resulting effective theory. In addition, we use it to demonstrate that the integration over the semi-fast modes does not deform the classical Yang-Mills equations of motion, thus validating a key assumption in the usual procedure adopted when deriving the renormalization group equation governing the evolution with energy of the effective theory. As far as the latter are concerned, we finally prove that its functional form is common, and it is determined by symmetry arguments alone. The formal properties of these equations valid in different regimes and/or approximations (e.g., the JIMWLK equation and its BFKL limit) can be therefore derived in a unified setting within this algebraic approach.
\end{abstract}

\section{Introduction and motivation}

The physics of high gluon densities and gluon saturation is one of the subfields of Quantum Chromodynamics (QCD) which has continuously attracted much attention over the last years, both theoretically and experimentally. The initial interest was concentrated on electron-proton deep inelastic scattering, but recently it has been shifted to the study of

\footnotetext{
a e-mail: binosi@ectstar.eu

b e-mail: andrea.quadri@mi.infn.it

c e-mail: trianta@ectstar.eu
}

heavy ion collisions [1,2], as gluon saturation plays a critical role in describing the initial wavefunction of the colliding nuclei and the early stages of the collision toward thermalization.

The first QCD-based calculation at small- $x$, where by $x$ we refer to the longitudinal momentum fraction of a parton, resulted in the BFKL equation [3-5], which predicted a sharp rise of the gluon distribution in the limit of interest. After the seminal work [6] in which the idea of gluon saturation was introduced and its necessity was emphasized, and a complementary attempt [7] based on imposing unitarity constraints, various methods to address physics at small- $x$ were developed. Here we shall only deal with the Color Glass Condensate (CGC), a modern approach which is based on effective field theory (EFT) techniques for integrating out degrees of freedom, and provides a well-defined framework that can be used for phenomenological applications [8].

The main concepts of the CGC were already contained in the so-called McLerran-Venugopalan (MV) model $[9,10]$, which aimed at describing the gluon distribution at small- $x$ in a very large nucleus, that is, with atomic number $A \gg 1$. For transverse separations smaller than $1 / \Lambda_{\mathrm{QCD}}$ this model treats the $A \times N_{c}$ valence quarks as uncorrelated long-lived color sources for the emission of soft gluons. Due to the large number of nucleons, a strong coherent color field can be created that leads to the saturation of gluon occupation numbers which become of order $1 / \alpha_{s}$. Despite its simplicity, since it does not contain any quantum (chromo)dynamics, the MV model, or at least some refined version of it, is still a reliable model for providing the initial conditions at moderate values of $x$ in heavy ion collisions.

However, such dynamics is necessary in order to evolve the wavefunction of a hadron (or a nucleus) to arbitrarily small values of $x$. This is not such an easy task, as it requires the resummation of large longitudinal logarithms in a dense environment. This program was quite successful and resulted in a renormalization group (RG) equation, known as the 
JIMWLK equation ${ }^{1}[11-17]$. This is a functional equation for the evolution of a weight function $W[\rho]$ which determines all the correlations of the color sources $\rho$. For a certain observable, the scattering of a small color dipole off the CGC in the multi-color limit, it leads to the Balitsky-Kovchegov (BK) equation $[18,19]$, which is a closed non-linear evolution equation. The JIMWLK equation is of the FokkerPlanck type, as established in [17], and thus it has an equivalent Langevin formulation [17,20], which has been recently extended to address the problem of gluon correlations at different values of $x$ [21]. Significant understanding towards the solution to the JIMWLK equation has been achieved by now, both via numerical techniques [22,23] which are based on the Langevin description, and via semi-analytic methods [24-26] based on a well-formulated Gaussian (meanfield) approximation. Let us also note that various works have appeared after the final version of the equation was written, in which simpler derivations have been given, certain aspects have been clarified or the validity of the equation has been extended to larger kinematic regimes [27-36].

As said, the derivation of the JIMWLK equation is rather involved, since one has to resum longitudinally enhanced contributions in the presence of a potentially strong background field. Moreover, it requires a special blend of gauge choices, mostly based on physical intuition, where the background field is kept in the Coulomb gauge while the modes to be integrated over are in the lightcone gauge. Here we would like to use techniques which are exploiting the Becchi-Rouet-Stora-Tyutin (BRST) symmetry and the associated (extended) Slavnov-Taylor (ST) identity in the presence of a non-trivial background [3739], in order to disentangle the gauge-dependent effects, due to the specific gauge choice adopted, from the gaugeinvariant physical quantities of the EFT. In particular, we will show that gauge invariance of the CGC effective action holds as a consequence of the fulfillment of the ST identity (after the integration of the semi-fast modes); hence, as expected on general physical grounds, the specific gauge used during the one-step quantum evolution is only a matter of convenience. ${ }^{2}$ We hasten to emphasize that this proof is not limited to the one-loop approximation, but it holds to all orders in the perturbative expansion on the semi-fast modes; in addition, being based on symmetry arguments only, it is regularization schemeindependent as well (as far as the ST identity is not broken).

\footnotetext{
1 The acronym stands for Jalilian-Marian, Iancu, McLerran, Weigert, Leonidov and Kovner.

${ }^{2}$ For example, if one uses a renormalizable gauge as opposed to the Light Cone gauge that is usually conveniently employed for the semifast modes in most of the CGC calculations, the derivation becomes more complicated (ghosts cannot be neglected anymore) but the final results are granted to coincide.
}

Moreover, one can also study how the background field equation of motion changes once quantum corrections are taken into account. Such an equation of motion is completely fixed by the ST identity in the presence of a non-trivial background and can be solved by a certain canonical transformation $[38,39]$. In the CGC approach the equation of motion for the background fast modes, valid after the one-step quantum evolution, is crucial in deriving the CGC evolution equations, since one must be able to prove that the updated background configuration can again be obtained by the same classical Yang-Mills equation, now in the presence of color charges with new correlators encoding the effects of the integration of the semi-fast gluons. That this is indeed the case is far from obvious, since, in general, the classical background equation of motion is not preserved once quantum corrections are taken into account [37]. Still, as we will show, in the CGC approach the clever choice of the (background) gauge and of the definition of the color charges [29] stabilizes the classical background equation of motion under radiative corrections, thus ensuring the formal consistency of the whole picture.

The structure of the paper is as follows. In Sect. 2 we review the CGC, paying particular attention to the physical motivation for constructing such an EFT and introducing the appropriate action and its symmetries. In Sect. 3 we discuss the BRST transformations of the various fields appearing in the action and in Sect. 4 we elaborate on the gauge-fixing term. In Sect. 5, which may be considered as the main section of the paper, we obtain the ST identity for the CGC effective action, generated upon the integration over the semi-fast modes introduced in Sect. 2. The ST identity imposes strong constraints on the CGC effective action for the soft gluon modes (in the presence of the fast background). Specifically, we will show that it is the key tool for establishing two important properties: (i) the gauge invariance of the effective action (irrespectively of the choice of the gauge fixing adopted in the integration of the semi-fast modes which is kept unspecified), to be discussed in Sect. 5.1, and (ii) the stability of the quantum-corrected equation of motion for the background derived in Sect. 5.2. In Sect. 6 we will indeed show that the classical field is not deformed by the one-step quantum evolution and that the classical relation between the background field and the color sources remains true after integrating the semi-fast modes. In Sect. 7 we show how the color charge correlations, generated from the quantum evolution, can be obtained from the effective action, leading to the CGC evolution equations. The general pattern of the derivation is dictated only by symmetry arguments, while the explicit expressions of the evolution Hamiltonian of course depends on the particular approximation used in the computation of the effective action. The conclusions are presented in Sect. 8, while the appendix contains a sketch of the derivation of the JIMWLK equation. 


\section{The color glass condensate}

A generic hadron ${ }^{3}$ in its rest frame is a rather complicated object. Its constituents are confined to live in the space occupied by the hadron under consideration and the typical time scale for the strong interactions among them is $1 / \Lambda_{\mathrm{QCD}}$, since there is no other scale in the problem. Thus, in general, one cannot say much without relying on non-perturbative methods.

The above description changes drastically when we go to the infinite momentum frame, a frame in which the hadron moves ultra-relativistically, usually along the $x^{3}$ direction by convention. Then hadronic time scales are dilated by a large Lorentz factor $\gamma$ and one has the possibility to separate calculable, but non-trivial, perturbative QCD dynamics from non-perturbative ones, as for example done in the standard analysis of electron-proton deep inelastic scattering.

At high-energy, by definition, we are interested in kinematics such that the hadron's longitudinal momentum is much larger than all possible transverse momenta, with the latter assumed to be larger than $\Lambda_{\mathrm{QCD}}$ so that we can rely on weak coupling techniques. For example, and in order to be more pragmatic, it has to be much larger than the transverse momenta of produced particles when collided with another hadron. In such a kinematic regime, a prominent role is played by the small- $x$ gluons, which are those gluons carrying a small fraction $x$ of the hadron's total longitudinal momentum.

QCD favors the generation of such small- $x$ gluons, since the emission of a gluon (but not that of a quark) with fraction $x$ from a parton (either quark or gluon) with fraction $x_{0}$ is proportional to $\bar{\alpha}_{s} \ln \left(x_{0} / x\right)$, with $\bar{\alpha}_{s}=\alpha_{s} N_{c} / \pi, \alpha_{s}=g^{2} / 4 \pi$ the QCD coupling and $N_{c}$ the number of colors. Clearly, in the limit of interest, the logarithm can overcome the smallness of $\bar{\alpha}_{s}$ and one needs to resum powers of $\bar{\alpha}_{s} \ln \left(x_{0} / x\right)$ to all orders in perturbation theory. This is equivalent to viewing this slowest gluon with fraction $x$ as being the lower end of a cascade composed of $n$ successive intermediate emissions of gluons with strongly ordered longitudinal momentum fractions, that is, with $x_{0} \gg x_{1} \gg \cdots \gg x_{n} \gg x$. On the contrary, transverse momenta are not ordered and therefore the transverse sizes of gluons remain typically the same in the course of evolution towards smaller values of $x$. Then the aforementioned resummation of the perturbative series leads to a fast, exponential in the rapidity $Y \equiv \ln (1 / x)$, increase in the gluon occupation number, i.e., in the number of gluons per unit phase space. This violates unitarity, since an occupation number should not be larger than $\sim 1 / \alpha_{s}$.

What has gone wrong in the above picture is that we have assumed small- $x$ gluons to be emitted independently from its

\footnotetext{
${ }^{3}$ Here we use the term hadron to also include the case of a nucleus with an arbitrary atomic number.
}

predecessors, an assumption which is well justified so long as the wavefunction is still dilute and naturally leads to exponentiation. However, this is not true any more when occupation numbers grow large. Then the emission of a small- $x$ gluon is a coherent phenomenon as the gluon is subjected to live in a dense environment. This mechanism suppresses the emission rate which eventually saturates, leading to (marginal) saturation ${ }^{4}$ of the gluon occupation number consistent with field theoretical requirements.

The CGC is a modern effective field theory which encompasses the above description for the small- $x$ components of the ultra-relativistic hadronic wavefunction. "Color" stands for the fact that we deal with a Yang-Mills theory and "condensate" is for the occupation numbers which can reach values of order $1 / \alpha_{s}$. The characterization "glass" comes about because gluons with different longitudinal momenta have different lifetimes. To be more precise, let us first introduce the light-cone coordinates $x^{\mu}=\left(x^{+}, x^{-}, \boldsymbol{x}\right)$ with $x^{ \pm}=\left(x^{0} \pm x^{3}\right) / \sqrt{2}$ and $\boldsymbol{x}=\left(x^{1}, x^{2}\right)$. For our convenience later on, let us also define here the 3 -vector $\vec{x}=\left(x^{-}, \boldsymbol{x}\right)$. Then the lifetime of a gluon is $\tau \sim p^{+} / p^{2}=x P^{+} / p^{2}$, where $P^{+}$is the longitudinal momentum of the hadron, meaning that gluons with smaller- $x$ live shorter. Thus, a gluon with fraction $x$ sees all the partons from which it has been coherently emitted as static, i.e., as $x^{+}$-independent, color sources. Moreover, by the same token, since these sources have much larger longitudinal momenta, they also have much shorter longitudinal wavelengths and therefore they appear to the emitted gluon as sharply localized in $x^{-}$.

So, let us consider an arbitrary longitudinal scale $\Lambda$ (clearly not to be confused with $\Lambda_{\mathrm{QCD}}$ ). If we are interested in correlations of "slow" gluons with momenta $k^{+}$smaller, but not much smaller, than $\Lambda$, one can integrate all the QCD dynamics above $\Lambda$ and absorb them in the aforementioned "fast" static color sources with a charge density $\rho^{a}\left(x^{-}, \boldsymbol{x}\right)$. Of course, these color sources have, in principle, highly nontrivial correlations which can be conveniently summarized in the weight function $W_{\Lambda}[\rho]$, which is a functional probability distribution. These correlations are automatically transmitted in the correlations of gluons with momenta below, but not very far from, the scale $\Lambda$. Thus, recalling also that we are interested in potentially large occupation numbers, or equivalently large gauge fields of order $1 / g$, we see that our problem turns into a classical Yang-Mills theory in the presence of sources.

As said, the weight function $W_{\Lambda}[\rho]$ includes all the quantum dynamics, among which non-perturbative effects, so that eventually one will have to resort to a modeling of infrared physics. However, one can predict how $W_{\Lambda}[\rho]$ evolves, and the resulting evolution is perturbative, as we will explain

\footnotetext{
${ }^{4}$ More precisely, the gluon occupation number still grows, albeit very slowly, like $\sim \ln (1 / x)$.
} 
below. If we become interested in even lower momenta $p^{+} \sim b \Lambda$ with $b \ll 1$, then it becomes obvious that some of the modes which were soft, now become fast and have to be integrated in the sources. Thus, the correlations of these color sources get logarithmically enhanced contributions of order $\bar{\alpha}_{s} \ln \left(\Lambda / p^{+}\right) \simeq \bar{\alpha}_{s} \ln (1 / b)$, and if the whole construction scheme is correct, these contributions should be absorbed in a new weight function $W_{b \Lambda}[\rho]$.

Clearly, one may wonder why this calculation is infrared safe. The straightforward answer is that this is done at the leading logarithmic level and the QCD coupling is taken to be fixed. Still, this is not a satisfactory answer because if the evolution becomes sensitive to very small transverse momenta, then it is almost guaranteed that the next to leading calculation will suffer from infrared divergences. However, the saturation of occupation numbers simply says that there is a scale $Q_{s}$, called the saturation momentum (or saturation scale), below which the initial exponential growth in $Y=\ln (1 / x)$ is tamed. This scale, which is dynamically generated, is a perturbative one as it increases rapidly with $Y[6,40-43]$, which means that even modes with arbitrarily high $\boldsymbol{p}$ will saturate at sufficiently small- $x$. Therefore, $Q_{s}$ is the natural scale to set the value of the coupling and, moreover, saturation (in the form of non-linear terms in evolution equations) will cut potentially dangerous infrared contributions in the course of evolution.

After this introductory description we come to the level where we can formulate our problem. Let us start by writing the action of our theory which reads

$S_{\mathrm{CGC}}[A, \rho]=S_{\mathrm{YM}}[A]+S_{W}[A, \rho]$.

In the expression above $S_{\mathrm{YM}}$ is the Yang-Mills action

$S_{\mathrm{YM}}=-\int \mathrm{d}^{4} x \frac{1}{N_{c}} \operatorname{Tr}\left[F_{\mu \nu} F^{\mu \nu}\right]$,

where the field strength is given in matrix form by $F_{\mu \nu}=$ $F_{\mu \nu}^{a} T^{a}$, with $T^{a}$ the generators in the adjoint representation of the $\mathrm{SU}(N)$ group; in components we have $F_{\mu \nu}^{a}=\partial_{\mu} A_{v}^{a}-$ $\partial_{\nu} A_{\mu}^{a}+g f^{a b c} A_{\mu}^{b} A_{\nu}^{c}$ with $f^{a b c}$ the $\mathrm{SU}(N)$ structure constants.

The piece $S_{W}$ in Eq. (2.1) contains the interactions of the color source $\rho$ which stands for the plus component (the only non-vanishing one) of the 4-current associated with the fast sources. These sources couple to the $A^{-}$component of the gauge field and as a first attempt, one may guess that $S_{W}$ is proportional to $\rho^{a}(\vec{x}) A_{a}^{-}(x)$. Such a term though cannot be gauge invariant, and eventually one has to define the action on a Schwinger-Keldysh contour in the complex time plane. It is given by [14]

$S_{W}[A, \rho]=\frac{\mathrm{i}}{g N_{c}} \int \mathrm{d}^{3} \vec{x} \operatorname{Tr}\left[\rho(\vec{x}) W_{C}(\vec{x})\right]$,

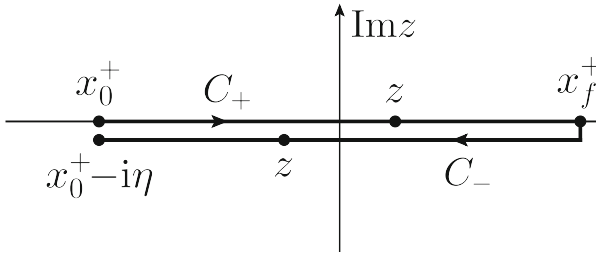

Fig. 1 The Schwinger-Keldysh contour in the complex time plane along which the source part of the action in Eq. (2.3) and the temporal Wilson line in Eq. (2.4) are defined

where $W_{C}(\vec{x})$ is the contour temporal Wilson line

$W_{C}(\vec{x})=\mathrm{T}_{C} \exp \left[\mathrm{i} g \int_{C} \mathrm{~d} z A^{-}(z, \vec{x})\right]$,

and as we shall show below it gives rise to the appropriate covariant equations of motion in the presence of a source. The contour $C=C_{+} \cup C_{-}$in Eqs. (2.3) and (2.4) is the aforementioned Schwinger-Keldysh contour defined as follows (cf. Fig. 1): $C_{+}$is the path along the real time axis, from $x_{0}^{+}$to $x_{f}^{+}$, while the points on $C_{-}$have a small imaginary part, that is, $z=x^{+}-\mathrm{i} \eta$ with $\eta \rightarrow 0_{+}$, and $x^{+}$runs backwards from $x_{f}^{+}$to $x_{0}^{+}$. Eventually we shall take the limits $x_{0}^{+} \rightarrow-\infty$ and $x_{f}^{+} \rightarrow+\infty$. In Eq. (2.4) $\mathrm{T}_{C}$ orders the matrices $A^{-}$from right to left as ones moves along the contour $C$, i.e., it coincides with chronological ordering along the $C_{+}$part of the contour and anti-chronological ordering along the $C_{-}$one. ${ }^{5}$

Let us now assume that $G(x) \in \mathrm{SU}(N)$ satisfies for any $\vec{x}$ the periodic condition

$G(-\infty-\mathrm{i} \eta, \vec{x})=G(-\infty, \vec{x})$.

Due to this property, one easily sees that the gauge transformations

$A^{\mu}(x) \mapsto G A^{\mu}(x) G^{\dagger}(x)+(\mathrm{i} / g) G(x) \partial^{\mu} G^{\dagger}(x)$,

$\rho(\vec{x}) \mapsto G(-\infty, \vec{x}) \rho(\vec{x}) G^{\dagger}(-\infty, \vec{x})$,

$W_{C}(\vec{x}) \mapsto G(-\infty-\mathrm{i} \eta, \vec{x}) W_{C}(\vec{x}) G^{\dagger}(-\infty, \vec{x})$,

leave the action $S_{W}[A, \rho]$ invariant. It is also instructive to notice that $S_{W}[A, \rho]$ may be equivalently written as

$S_{W}[A, \rho]=-\frac{1}{N_{c}} \int \mathrm{d}^{3} \vec{x} \int_{C} \mathrm{~d} z \operatorname{Tr}\left[\rho(\vec{x}) A^{-}(z, \vec{x}) W_{z,-\infty}(\vec{x})\right]$,

where the subscripts in the Wilson line simply mean that the contour integral in the complex time plane should now start at $-\infty$ (on the upper branch) and end at $z$ (either in

\footnotetext{
5 Notice that there is no issue regarding the contribution of the small vertical part at $x_{f}^{+}$to the Wilson line in Eq. (2.4); it just vanishes since $\eta \rightarrow 0$.
} 
the upper or in the lower branch). In order to show the equivalence between Eqs. (2.3) and (2.9) it appears easier to start from the latter. We see that $A^{-}(z, \vec{x}) W_{z,-\infty}(\vec{x})=$ $-(\mathrm{i} / g) \partial_{z} W_{z,-\infty}(\vec{x})$ and then we can trivially perform the $z$-integration (since the integrand is a total derivative) to arrive at Eq. (2.3). Notice that the lowest order term of the Wilson line in Eq. (2.9) leads to the linear (in the field) coupling proportional to $\rho^{a}(\vec{x}) A_{a}^{-}(x)$, while higher order terms restore the gauge invariance of the interaction.

Thus, having built a gauge invariant action, one is guaranteed to get the proper classical equations of motion in the presence of a color source, as we show now. Differentiating the action $S_{W}[A, \rho]$ in Eq. (2.3) with respect to (w.r.t.) $A_{a}^{-}$ (since this is the only component which couples to the source and thus modifies the classical Yang-Mills equations in the vacuum) successively we find

$$
\begin{aligned}
& T^{a} \frac{\delta S_{W}[A, \rho]}{\delta A_{a}^{-}(z, x)} \\
& \quad=-\frac{1}{N_{c}} T^{a} \operatorname{Tr}\left[\rho(\vec{x}) W_{-\infty-\mathrm{i} \eta, z}(\vec{x}) T^{a} W_{z,-\infty}(\vec{x})\right] \\
& \quad=-W_{x^{+},-\infty}(\vec{x}) \rho(\vec{x}) W_{x^{+},-\infty}^{\dagger}(\vec{x}) .
\end{aligned}
$$

In order to obtain the second inequality in the above, assume first that $z=x^{+}$, i.e. that it lies on $C_{+}$. (For $z=x^{+}-\mathrm{i} \eta$, that is, when it lies on $C_{-}$, the proof is totally analogous.) Then one can decompose one of the Wilson lines according to $W_{-\infty-\mathrm{i} \eta, x^{+}}=W_{-\infty-\mathrm{i} \eta, x^{+}-\mathrm{i} \eta} W_{x^{+}-\mathrm{i} \eta, \infty-\mathrm{i} \eta} W_{\infty, x^{+}}$. The last two evolution factors cancel each other, since at tree level $A^{-}$takes identical values on both sides of the contour for the same $x^{+}$, while the first factor can be rewritten as $W_{x^{+},-\infty}^{\dagger}$.

Thus, Eq. (2.10) together with the corresponding contributions from the Yang-Mills part of the action, lead to

$$
\mathcal{D}_{\nu}[A] F^{\nu \mu}(x)=\delta^{\mu+} W_{x^{+},-\infty}(x) \rho(\vec{x}) W_{x^{+},-\infty}^{\dagger}(x) \equiv J^{\mu}(x),
$$

where we have introduced the covariant derivative

$$
\mathcal{D}_{\nu}[A] \Phi=\partial_{\nu} \Phi-\mathrm{i} g[A, \Phi],
$$

for a generic matrix field $\Phi$. Thus, the r.h.s. of Eq. (2.11) means that the source $\rho$ is subjected to a color precession due to eikonal scattering off the time-dependent field $A^{-}$. This color precession is necessary in order to have covariant conservation of the current $J^{\mu}(x)$, that is, $\mathcal{D}_{\mu}[A] J^{\mu}(x)=0$.

In the absence of $A^{-}$, the source becomes $x^{+}$-independent, $F^{-i}$ (with $i=1,2$ ) vanishes automatically and it is possible to construct a solution with $F^{i j}=0$, that is, $A^{i}$ is a pure gauge. Then the only non-trivial field strength component is $F^{+-}$. Choosing a gauge finally fixes $A_{i}$, leaving us with only one independent degree of freedom, $A^{+}$; in particular, in the Coulomb gauge $\left(\partial_{i} A^{i}=0\right)$, the classical equation of motion reduces to the Poisson equation,

$-\nabla_{x}^{2} A^{+}(\vec{x})=\rho(\vec{x})$.

Then, by a gauge rotation, it is possible to obtain the classical solution in an arbitrary gauge.

Returning now to the quantum problem, one observes that the fact that we are in the infinite momentum frame makes possible to identify the quantum modes to be integrated out when performing one step in the quantum evolution, by splitting the gauge field $A^{\mu}$ into three pieces according to their support in momentum space. Specifically, we set

$A_{\mu}=\widehat{A}_{\mu}+a_{\mu}+\delta A_{\mu}$,

where (i) $\widehat{A}_{\mu}$ represents the fast modes with longitudinal momenta $\left|p^{+}\right|>\Lambda$ and is given by the classical solution to Eq. (2.11) in the absence of $A^{-}$and in an arbitrary gauge as has been described above, (ii) $a_{\mu}$ stands for the semi-fast modes, i.e., the modes with momenta $p^{+}$such that $\Lambda>\left|p^{+}\right|>b \Lambda$ (where we recall that $b \ll 1$ but with $\left.\bar{\alpha}_{s} \ln (1 / b) \ll 1\right)$ which will be integrated in the one-step quantum evolution, and (iii) $\delta A_{\mu}$ corresponds to the soft modes with momenta $\left|p^{+}\right|<b \Lambda$ which, like $\widehat{A}_{\mu}$, will be kept fixed during the quantum step.

\section{BRST transformations}

We now come to study the BRST symmetry of the action and the corresponding transformations of fields and sources. We will first start from the gluon and ghost sector, while later on we will focus on the classical color charge.

\subsection{Gluons}

When considering the total gauge field $A_{\mu}^{a}$, its corresponding BRST transformation coincides with the conventional gauge transformation in which the gauge parameter is replaced by the associated Faddeev-Popov ghost field $C^{a}$, that is, one has (with $s$ the BRST operator)

$s A_{\mu}^{a}=\mathcal{D}_{\mu}^{a b}[A] C^{b} ; \quad \mathcal{D}_{\mu}^{a b}[A]=\delta^{a b} \partial_{\mu}-g f^{a b c} A_{\mu}^{c}$,

where $s$ represents the BRST operator, and we have written the covariant derivative defined in Eq. (2.12) in component form. Next, for the background field $\widehat{A}_{\mu}^{a}$, one introduces the source $\Omega_{\mu}^{a}$ as its BRST doublet partner through ${ }^{6}$ [47-49]

$s \widehat{A}_{\mu}^{a}=\Omega_{\mu}^{a} ; \quad s \Omega_{\mu}^{a}=s^{2} \widehat{A}_{\mu}^{a}=0$.

\footnotetext{
${ }^{6}$ Briefly, a pair of variables $(u, v)$ such that $s u=v, s v=0$ is called a BRST doublet (with $v$ representing the BRST doublet partner of $u$ ). In ordinary perturbative quantum field theory, Eq. (3.2) implements the so-called doublet mechanism [44-46], preventing the background field from modifying the physical observables of the model.
} 
In the standard formulation of the background field method, where one has the decomposition $A_{\mu}^{a}=\widehat{A}_{\mu}^{a}+Q_{\mu}^{a}$, the transformations (3.2) and (3.1) would have allowed the unequivocal determination of the BRST transformation of the quantum field $Q_{\mu}^{a}$. However, in the present case there is an ambiguity due to the fact that the field $Q_{\mu}^{a}$ comprises two terms: the semi-fast modes $a_{\mu}^{a}$ (to be integrated over), and the soft modes $\delta A_{\mu}^{a}$.

It turns out that there is no way to disentangle the individual transformations of these two contributions other than by resorting to a physical argument of some kind. In this case the latter is provided by the fact that in order to preserve the BRST invariance of the action $S_{W}[\delta A, \rho], \delta A_{\mu}^{a}$ must clearly transform as a gauge connection.

In addition, since in the EFT spirit one is interested in the Green functions obtained after the semi-fast modes $a_{\mu}^{a}$ are integrated out, it is also natural to split the ghost field $C^{a}$ into a soft and a semi-fast component:

$C^{a}=c^{a}+\delta c^{a}$

where, as in the gauge field case, $\delta c^{a}$ denotes the soft modes and $c^{a}$ the semi-fast modes. Then one demands that

$s \delta A_{\mu}^{a}=\partial_{\mu} \delta c^{a}+g f^{a b c} \delta A_{\mu}^{b} \delta c^{c}$,

thus implementing the requirement that the soft field $\delta A_{\mu}^{a}$ transforms as a gauge connection w.r.t. the soft ghost $\delta c^{a}$.

At this point, the transformation of $a_{\mu}^{a}$ is fixed by the BRST variation of $A_{\mu}^{a}$ in Eq. (3.1) and by Eq. (3.2), once the splitting of the gluon and ghost fields of Eqs. (2.14) and (3.3) is imposed:

$$
\begin{aligned}
s a_{\mu}^{a}= & s A_{\mu}^{a}-s \delta A_{\mu}^{a}-s \widehat{A}_{\mu}^{a} \\
= & \partial_{\mu} c^{a}+g f^{a b c}\left(\widehat{A}_{\mu}^{b}+a_{\mu}^{b}+\delta A_{\mu}^{b}\right) c^{c} \\
& +g f^{a b c}\left(\widehat{A}_{\mu}^{b}+a_{\mu}^{b}\right) \delta c^{c}-\Omega_{\mu}^{a} .
\end{aligned}
$$

\subsection{Color charge}

In the presence of $\delta A^{-}$the current $J^{\mu}$ appearing on the r.h.s. of Eq. (2.11) is evaluated by using the temporal Wilson line from $z^{+} \rightarrow-\infty$ to $x^{+}$of the soft modes $\delta A^{-}$:

$W_{x^{+},-\infty}(x)=\mathrm{P} \exp \left[i g \int_{-\infty}^{x^{+}} \mathrm{d} z^{+} \delta A^{-}\left(z^{+}, \vec{x}\right)\right]$.

The current $J^{\mu}$ is then covariantly conserved and expresses the color precession of the static color charges in the presence of the time-dependent fields $\delta A^{-}$.

The classical solution $\widehat{A}_{\mu}^{a}$ is determined by the timedependent generalization of the solution in Eq. (2.13), that is, from

$\nabla_{x}^{2} \alpha(x)=-U^{\dagger}(x) J^{+}(x) U(x)$, with $J^{+}$the plus component of the color-rotated current in the r.h.s. of Eq. (2.11), and $U, U^{\dagger}$ the Wilson lines defined according to

$U^{\dagger}(x)=\mathrm{P} \exp \left[\mathrm{ig} \int_{-\infty}^{x^{-}} \mathrm{d} z^{-} \alpha\left(x^{+}, z^{-}, \boldsymbol{x}\right)\right]$,

where $\alpha(x)$ is to be identified with the only non-zero component of the classical background field in the Coulomb gauge, that is, $\alpha(x)=\widehat{A}^{+}(x)$.

It is most convenient to work directly with the color charge entering in the r.h.s. of Eq. (3.7) by setting

$\chi(x) \equiv U^{\dagger}(x) J^{+}(x) U(x)$.

Then $\chi$ becomes the independent variable and the original charge density $\rho=\rho(\chi)$ is determined by inverting Eq. (3.9) above; inserting the resulting expression in Eq. (2.9) yields finally $S_{W}[A, \chi]$. The BRST transformation of $\chi$ is easily derived after observing that by Eq. (3.9) $\chi$ transforms in the adjoint representation of $\mathrm{SU}(\mathrm{N})$, and therefore

$s \chi^{a}=g f^{a b c} \chi^{b} \delta c^{c}$.

Notice that the Wilson line $W$ in Eq. (3.6) only depends on $\delta A$ (and not on $a$ ), so that the BRST transformation in Eq. (3.10) contains only the soft ghosts $\delta c$.

To take into account the fluctuations in the color charge density $\chi^{a}$ induced by the integration over the semi-fast gluons $a$, we next split $\chi^{a}$ according to

$\chi^{a}=\widehat{\chi}^{a}+\delta \chi^{a}$.

Here, $\widehat{\chi}^{a}$ coincides with the current generating the classical configuration $\alpha$ in Eq. (3.7), and the corresponding BRST transformation can be therefore read off directly from its defining equation supplemented with Eq. (3.2):

$s \widehat{\chi}^{a}=-\nabla_{x}^{2} \Omega^{a+}(x)$.

The charge density $\delta \chi^{a}$ corresponds instead to that of the semi-fast gluons, and its BRST transformation can be finally obtained from the splitting (3.11)

$s \delta \chi^{a}(x)=s \chi^{a}(x)-s \widehat{\chi}^{a}(x)$,

with the r.h.s. determined by Eqs. (3.10) and (3.12). The important aspect to notice is that this transformation is independent of the soft gluon field $\delta A$.

\section{Gauge fixing}

The derivation of evolution equations such as the JIMWLK equation or its BFKL limit, requires to integrate out the semifast quantum fluctuations. It is at this point that the flexibility of working in the background field formulation of the theory 
manifests itself, as one has the freedom of choosing different gauge fixings for background and quantum fields (that is, the semi-fast and soft modes in the case at hand). In momentum space representation, this can be achieved by choosing a gauge-fixing functional of the type

$$
\begin{aligned}
\mathcal{F}^{a}(p)= & \theta\left(\left|p^{+}\right|-b \Lambda\right) \theta\left(\Lambda-\left|p^{+}\right|\right) \mathcal{F}_{\text {s.fast }}^{a}(p) \\
& +\theta\left(b \Lambda-\left|p^{+}\right|\right) \mathcal{F}_{\text {soft }}^{a}(p),
\end{aligned}
$$

where the semi-fast (soft) gauge-fixing function $\mathcal{F}_{\text {s.fast }}\left(\mathcal{F}_{\text {soft }}\right)$ depends only on the semi-fast (soft) gluon modes $a(\delta A)$. The resulting gauge-fixing action can then be calculated through the usual formula,

$S_{\mathrm{GF}+\mathrm{FPG}}=\int \mathrm{d}^{4} x s\left(\bar{C}^{a} \mathcal{F}^{a}+\frac{\xi}{2} \bar{C}^{a} B^{a}\right)$,

where $\xi$ is a gauge-fixing parameter, $\bar{C}^{a}$ the antighost field and $B^{a}$ the Nakanishi-Lautrup multiplier. Evidently, the splittings (2.14) and (3.3) induce a corresponding separation of these latter fields into their soft and semi-fast components according to

$\bar{C}^{a}=\bar{c}^{a}+\delta \bar{c}^{a} ; \quad B^{a}=b^{a}+\delta b^{a}$.

Due to the very simple form that the classical equation of motion assumes in the Coulomb gauge [see Eq. (2.13)] the soft gauge-fixing function will be chosen to be the Coulomb gauge one

$\mathcal{F}_{\text {soft }}^{a}(p)=-i p^{i} \delta A_{i}^{a}(p)$,

while the semi-fast function will be left, at the moment, unspecified. The ghost-dependent terms in (4.2) can be computed by using the BRST transformations in Eqs. (3.4) and (3.5); one obtains in this case

$$
\begin{aligned}
S_{\mathrm{FPG}}= & -\int \mathrm{d}^{4} p \theta\left(\left|p^{+}\right|-b \Lambda\right) \theta\left(\Lambda-\left|p^{+}\right|\right) \bar{c}^{a}(-p) s \mathcal{F}_{\mathrm{s.fast}}^{a}(p) \\
& -\int \mathrm{d}^{4} p \theta\left(b \Lambda-\left|p^{+}\right|\right) \delta \bar{c}^{a}(-p) p_{i}^{2} \delta c^{a}(p) \\
& +\int \mathrm{d}^{4} p \int \mathrm{d}^{4} q \theta\left(b \Lambda-\left|p^{+}\right|\right) g \\
& \times f^{a b c} \delta \bar{c}_{a}(-p) i p^{i} \delta A_{i}^{b}(q) \delta c^{c}(p-q) .
\end{aligned}
$$

\section{Slavnov-Taylor identity}

Until now our analysis has been strictly classical. In order to carry out the quantization of the theory, one needs a procedure to promote to the quantum level the non-linear symmetry generated by the BRST operator $s$. The most efficient way to accomplish this is through the introduction of certain external sources $\Phi^{*}$ (one for each field $\Phi$ transforming non-linearly under the symmetry being considered) which describe the renormalization of the composite operators that are bound to appear. These sources, called antifields [50], have opposite statistics with respect to the corresponding field $\Phi$, ghost charge $\operatorname{gh}\left(\Phi^{*}\right)=-1-\operatorname{gh}(\Phi)$, and, choosing the (mass) dimension of the Faddeev-Popov ghost fields to be zero, $\operatorname{dimension}{ }^{7} \operatorname{dim}\left(\Phi^{*}\right)=4-\operatorname{dim}(\Phi)$.

Antifields are then coupled to the composite operators generated by the BRST variation of the corresponding field through the term

$S_{\mathrm{AF}}=\int \mathrm{d}^{4} x \sum \Phi^{*}(x) s \Phi(x)$.

Then the invariance of the corresponding (background gauge-fixed) tree-level action $\Gamma^{(0)}$ under the BRST symmetry is encoded in the following ST identity:

$\mathcal{S} \Gamma^{(0)} \equiv \int \mathrm{d}^{4} z\left[\Omega_{\mu}^{a}(z) \frac{\delta \Gamma^{(0)}}{\delta \widehat{A}_{\mu}^{a}(z)}+\sum \frac{\delta \Gamma^{(0)}}{\delta \Phi^{*}(z)} \frac{\delta \Gamma^{(0)}}{\delta \Phi(z)}\right]=0$,

where the sum goes over all the fields of the model. If the theory is anomaly free, ${ }^{8}$ the same identity holds for the quantum vertex functional $\Gamma$ (that is, for the generator of the oneparticle irreducible amplitudes):

$\mathcal{S} \Gamma=0 ; \quad \Gamma=\Gamma\left[\Phi ; \widehat{A} ; \Phi^{*} ; \Omega\right]$.

In the CGC framework the procedure explained above is complicated by the fact that its tree-level action

$\Gamma^{(0)}=S_{\mathrm{CGC}}+S_{\mathrm{GF}}+S_{\mathrm{FPG}}+S_{\mathrm{AF}}$,

involves fields with support in different momentum regions, for which the corresponding antifields have to be defined. Specifically, the splittings of Eqs. (2.14) and (3.3) requires the introduction of the semi-fast antifields $a^{*}, c^{*}$ and the soft ones $\delta A^{*}, \delta c^{*}$. Thus, BRST invariance of the tree-level CGC action (5.4) is expressed through the ST identity

$$
\begin{aligned}
\mathcal{S} \Gamma^{(0)} \equiv & \int \mathrm{d}^{4} z\left[\Omega_{\mu}^{a}(z) \frac{\delta \Gamma^{(0)}}{\delta \widehat{A}_{\mu}^{a}(z)}+\frac{\delta \Gamma^{(0)}}{\delta a_{\mu}^{* a}(z)} \frac{\delta \Gamma^{(0)}}{\delta a_{\mu}^{a}(z)}\right. \\
& +\frac{\delta \Gamma^{(0)}}{\delta\left(\delta A_{\mu}^{* a}(z)\right)} \frac{\delta \Gamma^{(0)}}{\delta\left(\delta A_{\mu}^{a}(z)\right)} \\
& +\frac{\delta \Gamma^{(0)}}{\delta\left(\delta c^{* a}(z)\right)} \frac{\delta \Gamma^{(0)}}{\delta\left(\delta c^{a}(z)\right)}+\frac{\delta \Gamma^{(0)}}{\delta c^{* a}(z)} \frac{\delta \Gamma^{(0)}}{\delta c^{a}(z)} \\
& +b^{a}(z) \frac{\delta \Gamma^{(0)}}{\delta \bar{c}^{a}(z)}+\delta b^{a}(z) \frac{\delta \Gamma^{(0)}}{\delta\left(\delta \bar{c}^{a}(z)\right)} \\
& \left.+\frac{\delta \Gamma^{(0)}}{\delta\left(\delta \chi^{* a}(z)\right)} \frac{\delta \Gamma^{(0)}}{\delta\left(\delta \chi^{a}(z)\right)}\right]=0,
\end{aligned}
$$

\footnotetext{
7 These conventions ensure that the action has both ghost number as well as canonical dimension zero.

${ }^{8}$ Notice that the theory in the presence of the background is nonanomalous if and only if it is anomaly-free at zero background. This follows since, as already said, $\widehat{A}_{\mu}$ and $\Omega_{\mu}$ form a BRST doublet $[45,46]$ and hence they do not alter the cohomology of the gauge theory [45].
} 
which generalizes to the vertex functional $\Gamma$

$$
\begin{gathered}
\mathcal{S} \Gamma=0 ; \quad \Gamma=\Gamma[a, \delta A, c, \delta c, \bar{c}, \delta \bar{c}, b, \delta b, \delta \chi ; \widehat{A}, \\
\left.\widehat{\chi} ; a^{*}, \delta A^{*}, c^{*}, \delta c^{*} \delta \chi^{*} ; \Omega\right] .
\end{gathered}
$$

Notice that we have not introduced an antifield for $\bar{c}$ and $\delta \bar{c}$, since they transform linearly under the BRST operator. ${ }^{9}$

As has been previously explained, in the CGC framework, one is interested in the correlators of the quantum fluctuations $\delta \chi$ of the color charge density, once the semifast modes $a$ have been integrated out. Such correlators are therefore one-particle reducible w.r.t. all the semi-fast modes $(a, c, \bar{c}, b)$, and they are generated by a new effective action $\widetilde{\Gamma}\left[\delta A, \delta c, \delta \bar{c}, \delta b, \delta \chi ; \widehat{A}, \widehat{\chi} ; \delta A^{*}, \delta c^{*}, \delta \chi^{*} ; \Omega\right]$ satisfying an ST identity which differs from Eq. (5.5).

The effective action $\widetilde{\Gamma}$ can be formally obtained by starting from the connected generating functional $\mathcal{W}$, which is the Legendre transform of $\Gamma$ w.r.t. the different fields of the theory; one has

$$
\begin{aligned}
& \mathcal{W}=\Gamma+\int \mathrm{d}^{4} x \sum J_{\Phi} \Phi ; \quad J_{\Phi}=-(-1)^{\epsilon(\Phi)} \frac{\delta \Gamma}{\delta \Phi} ; \\
& \Phi=\frac{\delta \mathcal{W}}{\delta J_{\Phi}} ; \quad \frac{\delta \mathcal{W}}{\delta \Phi^{*}}=\frac{\delta \Gamma}{\delta \Phi^{*}}
\end{aligned}
$$

where $J_{\Phi}$ denotes the source of the quantum field $\Phi$, while $\epsilon(\Phi)$ represents the statistics of the field $\Phi$ (1 for anticommuting variables, 0 for commuting ones). Notice that $\Phi$ runs on all the quantum fields, including both the soft and the semi-fast modes.

Then, in terms of the connected generating functional $\mathcal{W}$, the ST identity (5.5) reads

$$
\begin{aligned}
& \int \mathrm{d}^{4} z \Omega_{\mu}^{a}(z) \frac{\delta \mathcal{W}}{\delta \widehat{A}_{\mu}^{a}(z)} \\
& =\int \mathrm{d}^{4} z \frac{\delta \mathcal{W}}{\delta\left(\delta A_{\mu}^{* a}(z)\right)} J_{\delta A_{a}^{\mu}}(z)+\int \mathrm{d}^{4} z \frac{\delta \mathcal{W}}{\delta a_{\mu}^{* a}(z)} J_{a_{a}^{\mu}}(z) \\
& \quad-\int \mathrm{d}^{4} z \frac{\delta \mathcal{W}}{\delta\left(\delta c^{* a}(z)\right)} J_{\delta c^{a}}(z)-\int \mathrm{d}^{4} z \frac{\delta \mathcal{W}}{\delta c^{* a}(z)} J_{c^{a}}(z) \\
& \quad-\int \mathrm{d}^{4} z \frac{\delta \mathcal{W}}{\delta J_{\delta b^{a}}(z)} J_{\delta \bar{c}^{a}}(z)-\int \mathrm{d}^{4} z \frac{\delta \mathcal{W}}{\delta J_{b^{a}}(z)} J_{\bar{c}^{a}}(z) \\
& \quad+\int \mathrm{d}^{4} z \frac{\delta \mathcal{W}}{\delta\left(\delta \chi^{* a}(z)\right)} J_{\delta \chi^{a}}(z) .
\end{aligned}
$$

Next, we define $\widetilde{\Gamma}$ by setting to zero each source associated to the fields we want to integrate out; this amounts to imposing their equation of motion and, diagrammatically, to consider amplitudes that are one-particle reducible (1-PR) w.r.t. such fields. We then obtain

\footnotetext{
9 The situation is similar to the one discussed in the case of the pair $(\widehat{A}, \Omega):(\bar{c}, b)$ and $(\delta \bar{c}, \delta b)$ form BRST doublets, and no antifield is necessary.
}

$$
\begin{aligned}
\widetilde{\Gamma}= & \left.\mathcal{W}\right|_{J_{a}=J_{c}=J_{\bar{c}}=J_{b}=0}+\int \mathrm{d}^{4} z J_{\delta A_{a}^{\mu}}(z) \delta A_{a}^{\mu}(z) \\
& +\int \mathrm{d}^{4} z J_{\delta \chi^{a}}(z) \delta \chi^{a}(z)+\int \mathrm{d}^{4} z J_{\delta c^{a}}(z) \delta c^{a}(z) \\
& +\int \mathrm{d}^{4} z J_{\delta \bar{c}^{a}}(z) \delta \bar{c}^{a}(z)+\int \mathrm{d}^{4} z J_{\delta b^{a}}(z) \delta b^{a}(z) .
\end{aligned}
$$

Notice that as we are not taking the Legendre transform w.r.t. $a, c, \bar{c}$ and $b, \widetilde{\Gamma}$ contains one-particle reducible diagrams with respect to these fields.

Finally, by setting $J_{a}=J_{c}=J_{\bar{c}}=J_{b}=0$ in Eq. (5.8) and performing afterwards the Legendre transform (5.9), one finds the modified ST identity

$$
\begin{aligned}
\mathcal{S} \widetilde{\Gamma} \equiv & \int \mathrm{d}^{4} z\left[\Omega_{\mu}^{a}(z) \frac{\delta \widetilde{\Gamma}}{\delta \widehat{A}_{\mu}^{a}(z)}+\frac{\delta \widetilde{\Gamma}}{\delta\left(\delta A_{\mu}^{* a}(z)\right)} \frac{\delta \widetilde{\Gamma}}{\delta\left(\delta A_{\mu}^{a}(z)\right)}\right. \\
& +\frac{\delta \widetilde{\Gamma}}{\delta\left(\delta c^{* a}(z)\right)} \frac{\delta \widetilde{\Gamma}}{\delta\left(\delta c^{a}(z)\right)}+\delta b^{a}(z) \frac{\delta \widetilde{\Gamma}}{\delta\left(\delta \bar{c}^{a}(z)\right)} \\
& \left.+\frac{\delta \widetilde{\Gamma}}{\delta\left(\delta \chi^{* a}(z)\right)} \frac{\delta \widetilde{\Gamma}}{\delta\left(\delta \chi^{a}(z)\right)}\right]=0
\end{aligned}
$$

Let us emphasize once again that even though this functional equation has the same form as the original ST identity in Eq. (5.2), amplitudes in $\widetilde{\Gamma}$ are not 1-PI w.r.t. the semi-fast modes. In addition, it holds provided that the classical CGC action (2.1) is gauge invariant; in particular, it does not rely on the first order expansion (eikonal approximation) of the piece $S_{W}$, describing the interaction of the color charges with the gluons.

Equation (5.10) has a rich physical meaning and expresses in compact form two important properties of the quantized theory: (i) as a consequence of the BRST symmetry associated with the $\mathrm{SU}(N)$ gauge invariance, at $\Omega_{\mu}=0$ one obtains the ST identity for the theory of the soft modes in the presence of a fast background; (ii) by taking a derivative w.r.t. $\Omega_{\mu}$ and then setting $\Omega_{\mu}=0$, one gets the quantumdeformed equation of motion for the background fast field $\widehat{A}_{\mu}$, which can be solved by a specific canonical transformation derived in $[38,39]$, allowing to reconstruct the full background dependence in the quantum theory. We will analyze each of these properties in more detail in the following two subsections.

\subsection{Gauge invariance}

By taking a derivative w.r.t. $\delta c$ of Eq. (5.10) and then setting $\delta c$ and $\Omega$, as well as $\delta b$, to zero, one finds

$$
\begin{gathered}
\int \mathrm{d}^{4} z\left[\frac{\delta^{2} \widetilde{\Gamma}}{\delta\left(\delta c_{b}(x)\right) \delta\left(\delta A_{\mu}^{* a}(z)\right)} \frac{\delta \widetilde{\Gamma}}{\delta\left(\delta A_{\mu}^{a}(z)\right)}\right. \\
\left.+\frac{\delta^{2} \widetilde{\Gamma}}{\delta\left(\delta c_{b}(x)\right) \delta\left(\delta \chi_{\mu}^{* a}(z)\right)} \frac{\delta \widetilde{\Gamma}}{\delta\left(\delta \chi_{\mu}^{a}(z)\right)}\right]=0 .
\end{gathered}
$$


We now notice that $\delta A^{*}$ is coupled to the BRST variation $s \delta$ of Eq. (3.4), while $\delta \chi^{*}$ couples to the BRST variation $s \delta \chi$ of Eq. (3.13); thus, neither of them is coupled (at $\Omega=0$ ) to $a$ and $c$. Since in $\widetilde{\Gamma}$ one does not integrate over $\delta A$ and $\delta c$, the Green functions involving one soft antifield remain classical, namely

$$
\begin{aligned}
& \frac{\delta^{2} \widetilde{\Gamma}}{\delta\left(\delta c_{b}(x)\right) \delta\left(\delta A_{\mu}^{* a}(z)\right)}=\frac{\delta^{2} \widetilde{\Gamma}^{(0)}}{\delta\left(\delta c_{b}(x)\right) \delta\left(\delta A_{\mu}^{* a}(z)\right)} \\
& =\delta^{a b} \partial_{\mu} \delta^{(4)}(x-z)+g f^{a c b} \delta A_{\mu}^{c} \delta^{(4)}(x-z), \\
& \left.\frac{\delta^{2} \widetilde{\Gamma}}{\delta\left(\delta c_{b}(x)\right) \delta\left(\delta \chi^{* a}(z)\right)}\right|_{\Omega=0}=\left.\frac{\delta^{2} \widetilde{\Gamma}^{(0)}}{\delta\left(\delta c_{b}(x)\right) \delta\left(\delta \chi^{* a}(z)\right)}\right|_{\Omega=0} \\
& \quad=g f^{a c b}\left[\widehat{\chi}^{c}(x)+\delta \chi^{c}(x)\right] \delta^{(4)}(x-z) .
\end{aligned}
$$

Hence Eq. (5.11) amounts to the statement of gauge invariance of the effective action $\widetilde{\Gamma}$. It is very important to notice that this result holds irrespectively of the gauge choice for the semi-fast modes $\mathcal{F}_{\text {s.fast }}$ : that is, gauge invariance follows as a consequence of the ST identity (5.10) and of the decomposition between semi-fast and soft modes, once the appropriate BRST symmetry, induced by this splitting, is taken into account.

\subsection{Background (quantum-corrected) equations of motion}

The identity (5.10) can be further simplified if we restrict our attention to the dependence on the background field for amplitudes involving only external $\delta A$ and/or $\delta \chi$ legs. This is clearly the case we want to focus on, as the $\delta \chi$ correlators generated by $\widetilde{\Gamma}$ are those that will be eventually identified with the momenta of the updated weight function $W_{b \Lambda}[\chi]$, i.e., the original $W_{\Lambda}[\chi]$ after a one-step quantum evolution. Setting $J_{\delta c^{a}}=J_{\delta \bar{c}^{a}}=0$ in Eq. (5.8), and taking again the Legendre transform one gets in this case the reduced identity

$$
\begin{aligned}
\mathcal{S} \widetilde{\Gamma} \equiv & \int \mathrm{d}^{4} z\left[\Omega_{\mu}^{a}(z) \frac{\delta \widetilde{\Gamma}}{\delta \widehat{A}_{\mu}^{a}(z)}+\frac{\delta \widetilde{\Gamma}}{\delta\left(\delta A_{\mu}^{* a}(z)\right)} \frac{\delta \widetilde{\Gamma}}{\delta\left(\delta A_{\mu}^{a}(z)\right)}\right. \\
& \left.+\frac{\delta \widetilde{\Gamma}}{\delta\left(\delta \chi^{* a}(z)\right)} \frac{\delta \widetilde{\Gamma}}{\delta\left(\delta \chi^{a}(z)\right)}\right]=0
\end{aligned}
$$

Taking a functional differentiation of Eq. (5.13) w.r.t. the source $\Omega$, and setting $\Omega=0$ afterwards, yields

$$
\begin{aligned}
\frac{\delta \widetilde{\Gamma}}{\delta \widehat{A}_{\mu}^{a}(x)}= & -\int \mathrm{d}^{4} z\left[\frac{\delta \widetilde{\Gamma}}{\delta \Omega_{\mu}^{a}(x) \delta\left(\delta A_{\nu}^{* b}(z)\right)} \frac{\delta \widetilde{\Gamma}}{\delta\left(\delta A_{\mu}^{a}(z)\right)}\right. \\
& \left.+\frac{\delta \widetilde{\Gamma}}{\delta \Omega_{\mu}^{a}(x) \delta\left(\delta \chi^{* b}(z)\right)} \frac{\delta \widetilde{\Gamma}}{\delta\left(\delta \chi^{b}(z)\right)}\right] .
\end{aligned}
$$

This is a fundamental equation for the EFT at hand, as it encodes how quantum fluctuations will modify the classical equation of motion (2.11). Such knowledge is evidently

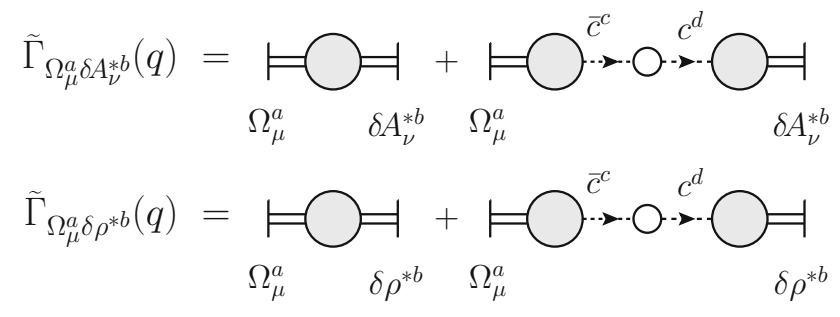

Fig. 2 The deformation functions $\widetilde{\Gamma}_{\Omega \delta A^{*}}(q)$ and $\widetilde{\Gamma}_{\Omega \delta \rho^{*}}(q)$. As these functions are 1-PR w.r.t. the semi-fast fields, on top of the 1-PI diagram one finds a 1-PR contributions proportional to the ghost propagator

required in order to be able to reconstruct the gluon fields generated by the new color charge density.

Indeed, the first term in Eq. (5.14) controls the (gaugedependent) deformation of the classical background-quantum splitting (2.14) induced by quantum corrections [37-39]; the second term fixes instead the functional dependence of the background $\widehat{A}$ on the color charge density $\delta \chi$, once quantum corrections are taken into account. This result is completely general, for it does not rely on the specific form of the action chosen, the gauge fixing adopted for the semi-fast modes, or even the order of approximation used while carrying out the one-step quantum evolution.

\section{Gauge invariant analysis of the deformation functions}

Consider now the deformation functions $\widetilde{\Gamma}_{\Omega \delta A^{*}}$ and $\widetilde{\Gamma}_{\Omega} \delta \chi^{*}$ explicitly appearing in Eq. (5.14), defined by

$\begin{aligned} \widetilde{\Gamma}_{\Omega_{\mu}^{a}(x) \delta A_{\nu}^{b *}(y)} & \equiv \frac{\delta^{2} \widetilde{\Gamma}}{\delta \Omega_{\mu}^{a}(x) \delta\left(\delta A_{\nu}^{b *}(y)\right)}, \\ \widetilde{\Gamma}_{\Omega_{\mu}^{a}(x) \delta \chi^{b *}(y)} & \equiv \frac{\delta^{2} \widetilde{\Gamma}}{\delta \Omega_{\mu}^{a}(x) \delta \chi^{b *}(y)},\end{aligned}$

and let us analyze their behavior.

To begin with observe that, as shown in Fig. 2, due to the quantum numbers of the source $\Omega$ and the antifields $\delta A^{*}$ and $\delta \chi^{*}$ there are only two contributions: the 1-PI term and a 1-PR graph in which the semi-fast ghosts are exchanged. In addition, while the couplings of the source $\Omega$ depend on the BRST variation of the (semi-fast) gauge fixing $s \mathcal{F}_{\text {s.fast }}$, the antifields $\delta A^{*}$ and $\delta \chi^{*}$ couple only to the BRST variations of the corresponding fields, which, in turn, do not depend on the choice of $\mathcal{F}_{\text {s.fast }}$, being fixed by symmetry requirements only.

This being said, let us start with $\widetilde{\Gamma}_{\Omega \delta A^{*}}$. As shown in Eq. (3.4) the antifield $\delta A^{*}$ couples at most to a soft ghost and a soft gluon. As $\widetilde{\Gamma}$ is constructed by integrating out the semi-fast modes (so that there cannot be internal soft lines in the $\widetilde{\Gamma}$-amplitudes), then both the 1-PI and the 1-PR terms 
are zero. Thus one is led to the result

$\widetilde{\Gamma}_{\Omega_{a}^{\mu} \delta A_{b}^{* v}}(x, y)=0$,

and, since at no point we have assumed the background field to be zero, taking any number $n$ of functional derivatives w.r.t. the background field yields

$\widetilde{\Gamma}_{\Omega_{a}^{\mu} \delta A_{b}^{* v}} \widehat{A}_{c_{1}}^{\rho_{1} \ldots \widehat{A}_{c_{n}}^{\rho_{n}}}\left(x, y, z_{1}, \ldots, z_{n}\right)=0$.

The analysis of the deformation function $\widetilde{\Gamma}_{\Omega} \delta \chi^{*}$ is similar. Again one observes that the couplings of the antifield $\delta \chi^{*}$ are dictated by the BRST variation (3.13) alone; that shows in turn that there are no couplings to any semi-fast mode. Thus again the 1-PR term is zero while the 1-PI diagram is confined at tree level, as, contrary to the previous case, Eq. (3.12) generates a tree-level coupling $\Omega \delta \chi^{*}$. Thus one finds

$\widetilde{\Gamma}_{\Omega_{a}^{\mu} \delta \chi_{b}^{*}}(x, y)=\Gamma_{\Omega_{a}^{\mu} \delta \chi_{b}^{*}}^{(0)}(x, y)=-\delta^{\mu+} \nabla_{x}^{2} \delta^{(4)}(x-y)$,

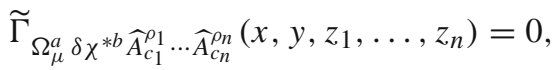

with the second relation obtained from the first one by taking $n$ functional differentiations w.r.t. the background field. Notice that the time dependence, induced by the Wilson line involving $\delta A^{-}$, has been reabsorbed into the definition of the $\chi$ charge in Eq. (3.9), thus leading to Eqs. (6.4).

Once again, the results established in Eqs. (6.2), (6.3), and (6.4) are independent from the form of the semi-fast gaugefixing function $\mathcal{F}_{\text {s.fast }}$, as for deriving them we never had to resort to any special property of the $\Omega$ source couplings. Thus the vanishing of the deformation function $\widetilde{\Gamma}_{\Omega \delta A^{*}}$ and the fact that $\widetilde{\Gamma}_{\Omega \delta x^{*}}$ is confined at tree level represent gauge invariant statements. As a result, one finds that, independently of the semi-fast gauge fixing for the $a$ modes, Eq. (5.14) further simplifies to

$\frac{\delta \widetilde{\Gamma}}{\delta \widehat{A}_{\mu}^{a}(x)}=-\int \mathrm{d}^{4} z \frac{\delta \widetilde{\Gamma}^{(0)}}{\delta \Omega_{\mu}^{a}(x) \delta\left(\delta \chi^{* b}(z)\right)} \frac{\delta \widetilde{\Gamma}}{\delta\left(\delta \chi^{b}(z)\right)}$.

This latter equation represents the full equation of motion for the background field $\widehat{A}$ when the semi-fast quantum fluctuations are integrated out; we once again stress that the deformation function $\Gamma_{\Omega} \delta \chi^{*}$ remains completely classical and background independent.

\subsection{Physical consequences}

The vanishing of the $\widetilde{\Gamma}_{\Omega \delta A^{*}}$ deformation function (together with all its background field derivatives) implies that the classical background-quantum splitting in Eq. (2.14) is not deformed after the one-step quantum evolution. Therefore the identification of $\delta A$ with the soft mode and of $\widehat{A}$ with the fast component of the gluon field is not spoiled by the quantum evolution.
This also clarifies an important conceptual point in the consistency of the separation of gluon modes carried out in the CGC framework. Indeed, the expansion of the pathintegral over the semi-fast modes [see Eq. (9.1)] is not performed around a stationary point of the action, as $\widehat{A}+\delta A$ is not a solution of the classical Yang-Mills equation of motion (2.11). Therefore in general one expects a shift, induced by quantum corrections, of the classical field configuration. However, such a shift would be proportional to $\widetilde{\Gamma}_{\Omega \delta A^{*}}[37-39]$ and therefore is absent in the CGC effective field theory.

Finally, Eq. (6.5) yields a relation between the correlators of the quantum fluctuations $\delta \chi$ of the color charge density and the background field $\widehat{A}$ once the semi-fast modes $a$ have been integrated out. Consider for example the case of the twopoint background sector. By taking first a derivative w.r.t. $\widehat{A}$ and then w.r.t. $\delta \chi$ Eq. (6.5) gives

$$
\begin{aligned}
& \widetilde{\Gamma}_{\widehat{A}_{a}^{\mu} \widehat{A}_{b}^{v}}\left(x_{1}, x_{2}\right)=-\int \mathrm{d}^{4} z \widetilde{\Gamma}_{\Omega_{a}^{\mu} \delta \chi_{c}^{*}}^{(0)}\left(x_{1}, z\right) \widetilde{\Gamma}_{\widehat{A}_{b}^{v} \delta \chi_{d}}\left(x_{2}, z\right), \\
& \widetilde{\Gamma}_{\widehat{A}_{a}^{\mu} \delta \chi_{b}}\left(x_{1}, x_{2}\right)=-\int \mathrm{d}^{4} z \widetilde{\Gamma}_{\Omega_{a}^{\mu} \delta \chi_{c}^{*}}^{(0)}\left(x_{1}, z\right) \widetilde{\Gamma}_{\delta x_{b} \delta \chi_{d}}\left(x_{2}, z\right),
\end{aligned}
$$

where all the two-point functions are to be evaluated at nonzero background. Substituting the second equation into the first one gives the condition

$$
\begin{aligned}
\widetilde{\Gamma}_{\widehat{A}_{a}^{\mu} \widehat{A}_{b}^{v}}\left(x_{1}, x_{2}\right)= & \int \mathrm{d}^{4} y \int \mathrm{d}^{4} z \widetilde{\Gamma}_{\Omega_{a}^{\mu} \delta \chi_{c}^{*}}^{(0)}\left(x_{1}, y\right) \widetilde{\Gamma}_{\Omega_{b}^{\nu} \delta \chi_{d}^{*}}^{(0)}\left(x_{2}, z\right) \\
& \times \widetilde{\Gamma}_{\delta \chi^{c} \delta \chi^{d}}(y, z) .
\end{aligned}
$$

By using Eq. (6.4) one therefore obtains the final relation (recall that $\alpha \equiv A^{+}$)

$$
\begin{aligned}
\widetilde{\Gamma}_{\alpha^{a} \alpha^{b}}\left(x_{1}, x_{2}\right)= & \int \mathrm{d}^{4} y \int \mathrm{d}^{4} z \nabla_{\boldsymbol{x}_{1}}^{2} \delta\left(x_{1}-y\right) \nabla_{\boldsymbol{x}_{2}}^{2} \delta\left(x_{2}-y\right) \\
& \times \widetilde{\Gamma}_{\delta \chi^{a} \delta \chi^{b}}(y, z) .
\end{aligned}
$$

It is instructive to study Eq. (6.8) at tree level and compare it with the relations between the correlators fixing the initial conditions for the CGC evolution. By projecting Eq. (6.8) at order zero in the loop expansion one finds

$$
\begin{aligned}
& \widetilde{\Gamma}_{\alpha^{a} \alpha^{b}}^{(0)}\left(x_{1}, x_{2}\right) \\
& \quad=\int \mathrm{d}^{4} y \int \mathrm{d}^{4} z \nabla_{x_{1}}^{2} \delta\left(x_{1}-y\right) \nabla_{x_{2}}^{2} \delta\left(x_{2}-y\right) \widetilde{\Gamma}_{\delta \chi^{a} \delta \chi^{b}}^{(0)}(y, z) .
\end{aligned}
$$

We take now, as a concrete example, a weight function corresponding to the simple Gaussian of the MV model [51] for a large nucleus with atomic number $A \gg 1$ :

$W_{A}[\chi]=\mathcal{N} \exp \left\{-\frac{1}{2} \int \mathrm{d}^{3} x \frac{\chi^{a}(\vec{x}) \chi^{a}(\vec{x})}{\lambda_{A}\left(x^{-}\right)}\right\}$. 
In such a case, one has [51]

$\left\langle\alpha^{a}(\vec{x}) \alpha^{b}(\vec{y})\right\rangle_{A}=\delta^{a b} \delta\left(x^{-}-y^{-}\right) \gamma_{A}\left(x^{-}, x_{\perp}-y_{\perp}\right) ;$

$\gamma_{A}\left(x^{-}, k_{\perp}\right)=\frac{1}{k_{\perp}^{4}} \lambda_{A}\left(x^{-}\right)$.

Hence, one can identify the inverse of the propagator $\left\langle\alpha^{a} \alpha^{b}\right\rangle_{A}^{-1}$ with the two-point function $\widetilde{\Gamma}_{\alpha^{a} \alpha^{b}}^{(0)}$ and similarly, upon splitting the field $\chi=\widehat{\chi}+\delta \chi$ as custom in the background field method, $\lambda_{A}^{-1}\left(x^{-}\right)$with $\widetilde{\Gamma}_{\delta \chi \delta \chi}^{(0)}$. Since the splitting of the color sources is trivially given by (3.11), we arrive at the natural identification

$W_{A}[\chi]=\mathcal{N} \exp \{-\widetilde{\Gamma}[\chi]\}$,

where it is understood that the r.h.s. is evaluated at zero gauge fields (we only look at the correlators of the color sources).

Let us now project Eq. (6.8) at the one-loop level i.e., at the level of approximation of the CGC, that is $\alpha_{s} \ln 1 / b$. An obvious extension of the classical analysis just carried out yields the identifications

$$
\begin{aligned}
& \tilde{\rho} \leftrightarrow \widehat{\chi} ; \quad \delta \widetilde{\rho} \leftrightarrow \delta \chi ; \quad\langle\mathrm{T}[\delta \alpha \delta \alpha]\rangle^{-1} \leftrightarrow \widetilde{\Gamma}_{\alpha \alpha}^{(1)} \\
& \quad\langle\mathrm{T}[\delta \tilde{\rho} \delta \widetilde{\rho}]\rangle^{-1} \leftrightarrow \widetilde{\Gamma}_{\delta \chi \delta \chi}^{(1)},
\end{aligned}
$$

where $\delta \widetilde{\rho}$ is the induced color charge density of the semi-fast gluons. Then, by inverting Eq. (6.8), one obtains the relation between the correlators of the background fields and those of $\delta \tilde{\rho}$, namely

$$
\begin{aligned}
\left\langle\mathrm{T}\left[\delta \alpha^{a}\left(x_{1}\right) \delta \alpha^{b}\left(x_{2}\right)\right]\right\rangle= & \int \mathrm{d}^{4} y \int \mathrm{d}^{4} z \boldsymbol{\Delta}\left(x_{1}-y\right) \boldsymbol{\Delta}\left(x_{2}-y\right) \\
& \left\langle\mathrm{T}\left[\delta \widetilde{\rho}^{a}(y) \delta \widetilde{\rho}^{b}(z)\right]\right\rangle,
\end{aligned}
$$

with $\boldsymbol{\Delta}(x-y)$ the inverse of the Laplacian operator in two dimensions. Thus, the relationship between the gauge field and the color sources remains classical, even though the correlations of these sources are given by a quantum computation.

\section{Evolution equations}

Equation (6.8) shows that, to all orders in the loop expansion, the relation between the background field and the color charge density remains classical. This in turn implies that the non-trivial effects of the one-step quantum evolution can be described by studying the evolution of the correlators of the color sources.

Within the framework we have introduced so far, one can obtain the JIMWLK equation through the (rather natural) requirement that the correlators of the induced color source $\delta \widetilde{\rho}$ coincide with the correlators of the quantum fluctuations $\delta \chi$ in our quantum EFT. Setting $\tau=\alpha_{s} \ln 1 / b$ one has (we will suppress for the moment all color and Lorentz indices)

$$
\begin{aligned}
& \frac{\partial}{\partial \tau}\left\langle T\left[\delta \chi\left(x_{1}\right) \cdots \delta \chi\left(x_{n}\right)\right]\right\rangle \\
& \quad=\frac{\partial}{\partial \tau} \int \mathcal{D}[\delta \chi] \delta \chi\left(x_{1}\right) \cdots \delta \chi\left(x_{n}\right) \exp \left[\tau \Delta S_{\text {eff }}+\cdots\right] \\
& \quad=\int \mathcal{D}[\delta \chi] \delta \chi\left(x_{1}\right) \cdots \delta \chi\left(x_{n}\right) \Delta S_{\text {eff }} \exp \left[\tau \Delta S_{\text {eff }}+\cdots\right],
\end{aligned}
$$

where the dots indicate $\tau$-independent terms, and one has used the fact that the $\tau$ dependence at leading logarithmic order is a factor in front of $\Delta S_{\text {eff }}$. The latter is certainly true when calculating the effective action with the undressed gluon propagator; moreover, from the analysis given in the appendix we see that this will be true even when using the background dressed propagator.

Consider now the expansion of the effective action at $\delta A^{-}=0$; one has

$$
\begin{aligned}
\Delta S_{\mathrm{eff}}= & \sum_{m=0}^{\infty} \frac{1}{m !} \int_{y} \underbrace{\frac{\delta^{m} \Delta S_{\mathrm{eff}}}{\delta\left(\delta A^{-}\left(y_{1}\right)\right) \cdots \delta\left(\delta A^{-}\left(y_{m}\right)\right)}}_{\Gamma_{m}\left(y_{1}, \ldots, y_{m}\right)} \\
& \times \delta A^{-}\left(y_{1}\right) \cdots \delta A^{-}\left(y_{m}\right),
\end{aligned}
$$

where we have defined $\int_{y}=\int \mathrm{d}^{4} y_{1} \cdots \int \mathrm{d}^{4} y_{m}$; notice, in addition, that the coefficient functions $\Gamma_{m}\left(y_{1}, \ldots, y_{m}\right)$ are background-dependent.

At the relevant order in the eikonal approximation $\delta \chi$ is coupled to $\delta A^{-}$through a bilinear vertex in $S_{W}$ in Eq. (2.9). Therefore, at this order of approximation, in the CGC effective field theory (where no quantum integration is carried out over $\delta A^{-}$), each external $\delta A^{-}$leg can be converted into a $\delta \chi$ leg. Physically this means that $\delta A^{-}$plays the role of the source of $\delta \chi$. By taking this fact into account the expansion in the r.h.s. of Eq. (7.2) yields

$$
\begin{aligned}
& \frac{\partial}{\partial \tau}\left\langle T\left[\delta \chi\left(x_{1}\right) \cdots \delta \chi\left(x_{n}\right)\right]\right\rangle \\
& \quad=\sum_{m=0}^{\infty} \frac{1}{m !} \int_{y} \Gamma_{m}\left(y_{1}, \ldots, y_{m}\right) \\
& \quad \int \mathcal{D}[\delta \chi] \delta \chi\left(x_{1}\right) \cdots \delta \chi\left(x_{n}\right) \delta \chi\left(y_{1}\right) \cdots \delta \chi\left(y_{m}\right) \\
& \quad \times \exp \left[\tau \Delta S_{\text {eff }}+\cdots\right] .
\end{aligned}
$$

Let us next introduce the notation

$$
\begin{aligned}
& \mathcal{G}^{n ; m}\left(x_{1}, \ldots, x_{n} ; y_{1}, \ldots, y_{m}\right) \\
& \quad=\left\langle T \delta \chi\left(x_{1}\right) \cdots \delta \chi\left(x_{n}\right) \delta \chi\left(y_{1}\right) \cdots \delta \chi\left(y_{m}\right)\right\rangle,
\end{aligned}
$$

so that the previous equation reads

$$
\begin{aligned}
& \frac{\partial}{\partial \tau} \mathcal{G}^{n ; 0}\left(x_{1}, \ldots, x_{n}\right)=\sum_{m=0}^{\infty} \frac{1}{m !} \int_{y} \Gamma_{m}\left(y_{1}, \ldots, y_{m}\right) \\
& \times \mathcal{G}^{n ; m}\left(x_{1}, \ldots, x_{n} ; y_{1}, \ldots, y_{m}\right) .
\end{aligned}
$$


Now, recall that the field $\delta \chi$ describes the fluctuations of the charge density induced by quantum corrections; therefore the corresponding correlators will be associated to the momenta of the classical probability distribution (which incorporates semi-fast quantum corrections). Thus one has the identification

$$
\begin{aligned}
& \mathcal{G}^{n ; m}\left(x_{1}, \ldots, x_{n} ; y_{1}, \ldots, y_{m}\right) \\
& \equiv \frac{\delta^{n+m} W}{\delta(\delta \chi)\left(x_{1}\right) \cdots \delta(\delta \chi)\left(x_{n}\right) \delta(\delta \chi)\left(y_{1}\right) \cdots \delta(\delta \chi)\left(y_{m}\right)},
\end{aligned}
$$

so that Eq. (7.5) will read

$$
\begin{aligned}
& \frac{\partial}{\partial \tau} \frac{\delta^{n} W}{\delta(\delta \chi)\left(x_{1}\right) \cdots \delta(\delta \chi)\left(x_{n}\right)}=\sum_{m=0}^{\infty} \frac{1}{m !} \int_{y} \Gamma_{m}\left(y_{1}, \ldots, y_{m}\right) \\
& \quad \times \frac{\delta^{n+m} W}{\delta(\delta \chi)\left(x_{1}\right) \cdots \delta(\delta \chi)\left(x_{n}\right) \delta(\delta \chi)\left(y_{1}\right) \cdots \delta(\delta \chi)\left(y_{m}\right)}
\end{aligned}
$$

which for $n=0$ gives the following evolution equation:

$$
\frac{\partial}{\partial \tau} W=\sum_{m=0}^{\infty} \frac{1}{m !} \int_{y} \Gamma_{m}\left(y_{1}, \ldots, y_{m}\right) \frac{\delta^{m} W}{\delta(\delta \chi)\left(y_{1}\right) \cdots \delta(\delta \chi)\left(y_{m}\right)} .
$$

The derivation of this equation relies only on the assumption that the $\tau$ dependence factors out; the form of $\Delta S_{\text {eff }}$ is not needed. Therefore Eq. (7.8) is valid for both the JIMWLK and its BFKL limit, the difference between the two cases being given by the form that the correlation functions $\Gamma_{m}\left(y_{1}, \ldots, y_{m}\right)$ assume.

By comparing Eq. (7.8) with Eq. (7.2) one gets back the well-known result that the effective Hamiltonian can be obtained by replacing $\delta A^{-}(x)$ in the effective action with the differential operator $\frac{\delta}{\delta(\delta x)(x)}$. However, a remark is in order here. In the eikonal approximation the couplings between $\delta A^{-}$and both $\widehat{\chi}$ and $\delta \chi$ are the same, since they are obtained from $\int \mathrm{d}^{4} x \delta A^{-}(x) \chi(x)$ after the splitting $\chi=\widehat{\chi}+\delta \chi$. Therefore one can safely replace everywhere in Eq. (7.8) $\delta \chi$ with $\widehat{\chi}$ and get the customary form of the Hamiltonian evolution for $W$. This is in agreement with the previously stated prescription that the Green functions of the classical $\widehat{\chi}$ source, generated by $W$, coincide with the correlators of the quantum fluctuations $\delta \chi$ in the effective field theory of the CGC. Notice, however, that in general one cannot dispose of $\delta \chi$ altogether (and work only with $\widehat{\chi}$ ), since $\delta \chi$ is required in order to formulate the reduced ST identity (5.13), which holds to all orders in the perturbative expansion.

What we have achieved here is separating the derivation of the general form of the evolution equation (which is dictated by the symmetries of the theory alone) from the calculation of $\Delta S_{\text {eff }}$, which is rather a result of the approxima- tions one would like to introduce due to the particular regime one is interested in. The detailed evaluation of $\Delta S_{\text {eff }}$ for the JIMWLK equation is given in the appendix.

\section{Conclusions}

In this paper we have clarified the role played by the fundamental BRST symmetry of the QCD action in constraining the form of the high-energy evolution equation the theory can give rise to. In particular, we have concentrated on the EFT of the CGC, and achieved a complete separation between the general features of evolution equations, which only depend on the symmetry content of the theory, from the specific aspects related, e.g., to the choice of the gauge for the semi-fast modes or the particular approximation used in the computation of the EFT (one-loop) action.

The crucial enabling step has been the identification of the correct BRST symmetry of the CGC theory holding after the gluon field has been separated into its fast, semi-fast, and soft components. Once this has been done, the corresponding ST identity encoding at the quantum level the (classical) BRST invariance of the action can be written down.

A plethora of results then naturally follows. To begin with, the gauge invariance of the EFT (after the one-step quantum evolution) is a direct consequence of the mere existence of this identity. As a second result, one is able to prove that the classical Yang-Mills equations of motion are not deformed by the quantum corrections induced when integrating out the semi-fast field $a$. This implies that the classical description of the CGC at the new scale $b \Lambda$ in terms of a modified weight function $W_{b \Lambda}[\rho]$, with the same equations of motion holding at the scale $\Lambda$, is indeed consistent. This is a crucial ingredient in the derivation of the evolution equations, and it is remarkable that it can be derived on the basis of symmetry arguments only. Finally, one can prove that the exact form of the evolution equation is determined by the BRST symmetry alone; the approximations made in the calculation of the effective action account instead for which of the various evolution equations known in the literature (e.g., the JIMWLK or its BFKL limit) one is using. It should be noticed that in deriving all the aforementioned results, at no point we have fixed the gauge for the semi-fast modes $a$, which, as a matter of fact, has been left unspecified. ${ }^{10}$

We believe that the methods introduced here might help in going beyond the approximations usually employed. For instance, it is not necessary to take the eikonal limit in order to write down the ST identity, and therefore it is very likely that one can go beyond this approximation (cf. [52]). In addition,

\footnotetext{
${ }^{10}$ It is, however, convenient in the CGC theory to fix the background Coulomb gauge for the fast background $\widehat{A}$, due to the particularly easy form that the background equation of motion assumes in this case.
} 
a more general evolution equation than the one presented in (7.8) can be derived by using algebraic techniques [44], thus dropping the assumption of linearity in $\tau$. Even though such matters have not been addressed here, they deserve further investigation.

Acknowledgments We would like to thank Edmond Iancu for useful and stimulating discussions.

Open Access This article is distributed under the terms of the Creative Commons Attribution License which permits any use, distribution, and reproduction in any medium, provided the original author(s) and the source are credited.

Funded by $\mathrm{SCOAP}^{3}$ / License Version CC BY 4.0.

\section{Appendix A: Effective action for the JIMWLK equation}

In this appendix we give a "sketch" of the derivation of the JIMWLK Hamiltonian. The total field $A_{\mu}$ in the CGC is split according to Eq. (2.14) and the goal is to construct an effective action $S_{\text {eff }}$ quadratic in $\delta A^{-}$and to all orders in $\widehat{A}_{\mu}$, by integrating the semi-fast modes $a_{\mu}$ having longitudinal momenta $k^{+}$such that $b \Lambda \ll\left|k^{+}\right| \ll \Lambda$. Therefore we define

$$
\exp \left(\mathrm{i} S_{\mathrm{eff}}\right)=\int_{b \Lambda}^{\Lambda} \mathcal{D} a \exp (\mathrm{i} S) .
$$

$S$ is the sum of the CGC action $S_{\mathrm{CGC}}$, given by Eq. (2.1), and the gauge-fixing and Faddeev-Popov part $S_{\mathrm{GF}+\mathrm{FPG}}$ in Eq. (4.2).

$S_{\text {CGC }}$ generates the classical field equations in the presence of a static source $\rho(\vec{x})$ when $\delta A^{\mu}=0$, as in Eq. (2.11). The background field $\widehat{A}^{\mu}$ in Eq. (2.14) can be determined by the solution to the Poisson equation as we explain now. In the light-cone gauge only the transverse components are non-zero, i.e. $\widehat{A}_{\mu}=\delta_{\mu i} \widehat{A}_{i}$, and therefore we have

$\mathcal{D}_{\nu} F^{\nu+}=-\mathcal{D}_{i} \partial^{+} \widehat{A}^{i}=\rho(\vec{x})$.

Here we have made use of the fact that $F^{-+}=-\partial^{+} \delta A^{-} \simeq$ 0 , which comes about because $\delta A^{-}$contains modes with very small longitudinal momenta and thus its variation with $x^{-}$is very slow. The solution to the classical equation (9.2) is

$$
\widehat{A}^{i}=\frac{\mathrm{i}}{g} U(\vec{x}) \partial^{i} U^{\dagger}(\vec{x}),
$$

with the Wilson line $U^{\dagger}$ given by

$$
U^{\dagger}(\vec{x})=\mathrm{P} \exp \left[\mathrm{i} g \int_{-\infty}^{x^{-}} \mathrm{d} z^{-} \alpha^{a}\left(z^{-}, \boldsymbol{x}\right) T^{a}\right] .
$$

In the above we have defined $\alpha \equiv \widehat{A}^{+}$which is the only non-vanishing component of the background gauge field in the Coulomb gauge and satisfies

$\nabla_{x}^{2} \alpha(\vec{x})=-\chi(\vec{x})$,

with $\chi(\vec{x})$ the corresponding (static) source in the Coulomb gauge. Notice that in the JIMWLK approximation we set the $W$ rotation equal to 1 , because the rest of the calculation gives already the dominant $\left(\delta A^{-}\right)^{2}$ dependence.

The choice of the Coulomb gauge for the soft modes and of the light-cone gauge for the semi-fast ones uniquely fixes $S_{\mathrm{GF}+\mathrm{FPG}}$. Since in the light-cone gauge the ghosts decouple, they can be neglected while performing the one-step quantum evolution. Moreover, in order to derive the effective action required to obtain the JIMWLK Hamiltonian, the Green functions of the Nakanishi-Lautrup field $\delta b$ and of the soft ghosts and antighosts $\delta c, \delta \bar{c}$ are not needed and thus one can simply take $S=S_{\mathrm{CGC}}$.

Now we expand the action $S$ around $A_{0}^{\mu} \equiv \delta^{\mu i} \widehat{A}^{i}+$ $\delta^{\mu-} \delta A^{-}$and, in view of the Gaussian integration to follow, to second order in the semi-fast modes $a^{\mu}$, that is,

$S=S_{0}+\frac{\delta S}{\delta A^{i}} a^{i}+\frac{1}{2} a^{\mu} G_{\mu \nu}^{-1} a^{\nu}$.

It should be noticed that the expansion is not around the solution of the Yang-Mills equation of motion $\widehat{A}$, rather around $A_{0}$. I.e. one is not expanding around a stationary point of the action and thus, in general, one would expect that quantum corrections will deform the classical background solution. However, in the CGC this does not occur, as a consequence of the stability of the background equation of motion (6.5).

The propagator $G_{\mu \nu}$ is in the presence of only the background field $\widehat{A}^{i}$, since the field $\delta A^{-}$can be set equal to zero to the order of accuracy. The coefficient of the linear term may be written as

$$
\frac{\delta S}{\delta A^{i}}=\mathcal{D}_{\nu} F^{v i}=\mathcal{D}_{j} F^{j i}+\mathcal{D}^{+} F^{-i}+\mathcal{D}^{-} F^{+i} \simeq 2 \mathcal{D}^{+} F^{-i},
$$

where we have used the fact that $F^{i j}$ is a pure gauge, i.e. $F^{i j}=0$, and also the approximate equality $\mathcal{D}^{-} F^{+i} \simeq$ $\mathcal{D}^{+} F^{-i}$. The latter is due to the fact that $\partial^{+} \delta A^{-} \simeq 0$ as justified earlier. Now it becomes straightforward to perform the integration over the semi-fast modes $a^{\mu}$ to obtain the change of the effective action. It is given by a four-dimensional double integral, more precisely,

$$
\begin{aligned}
& \Delta S_{\text {eff }}=-\frac{1}{2} \int \mathrm{d}^{4} x \mathrm{~d}^{4} y\left[2 \mathcal{D}^{+} F^{-i}(x)\right] G^{i j}(x, y) \\
& \quad \times\left[2 \mathcal{D}^{+} F^{-i}(y)\right] .
\end{aligned}
$$

So far we have been working in the light-cone gauge, where ghost modes decouple and therefore can be neglected when integrating out the soft modes. In explicit computations this 
is a major simplification, which favors the light-cone gauge with respect to other gauge choices for the $a_{\mu}$ fields, in which ghosts do not decouple any more.

Since Eq. (9.8) has a gauge invariant form, it will be more convenient to calculate it in the Coulomb gauge. Even though the propagator is an important element of the calculation, since it also contains the logarithmic enhancement, here we shall just give its final form. It reads [29]

$$
\begin{aligned}
G^{i j}(x, y)= & -\frac{\mathrm{i} \Delta \tau}{4 \pi} \delta^{i j} \delta^{(2)}(\boldsymbol{x}-\boldsymbol{y})\left[\Theta\left(x^{-}-y^{-}\right) U_{x^{-} y^{-}}^{\dagger}(\boldsymbol{x})\right. \\
& \left.+\Theta\left(y^{-}-x^{-}\right) U_{y^{-} x^{-}}(\boldsymbol{x})\right],
\end{aligned}
$$

with $\Delta \tau=\ln (1 / b)$ representing as usual the differential enhancement in the longitudinal phase space and where the Wilson line $U^{\dagger}$ is given by a similar expression to the one in (9.4), but with the lower limit replaced by $y^{-}$. Using the fact that the propagator satisfies $\mathcal{D}^{+}(x) G^{i j}(x, y)=0$, it is not hard to show that the integrand in (9.8) is a total derivative with respect to both $x^{-}$and $y^{-}$and thus the integration is determined by the surface terms. Furthermore, the propagator is independent of the light-cone time and we can immediately integrate over $x^{+}$and $y^{+}$. Using $F^{-i}=-\partial^{i} \delta A^{-}$and defining for notational simplicity $A^{-}(\vec{x})=\int \mathrm{d} x^{+} \delta A^{-}(x)$ we arrive at

$$
\begin{aligned}
\frac{\Delta S_{\mathrm{eff}}}{\Delta \tau}= & \frac{\mathrm{i}}{2 \pi} \int \mathrm{d}^{2} \boldsymbol{x}\left[\partial^{i} A^{-}(\infty, \boldsymbol{x}) \partial^{i} A^{-}(\infty, \boldsymbol{x})\right. \\
& +\partial^{i} A^{-}(-\infty, \boldsymbol{x}) \partial^{i} A^{-}(-\infty, \boldsymbol{x}) \\
& -\partial^{i} A^{-}(\infty, \boldsymbol{x}) U^{\dagger}(\boldsymbol{x}) \partial^{i} A^{-}(-\infty, \boldsymbol{x}) \\
& \left.-\partial^{i} A^{-}(-\infty, \boldsymbol{x}) U(\boldsymbol{x}) \partial^{i} A^{-}(\infty, \boldsymbol{x})\right],
\end{aligned}
$$

where now the Wilson lines are as in Eq. (9.4)), but with the integration extending over the whole longitudinal axis. The analysis in Sect. 7 suggests that the evolution Hamiltonian can be obtained via the replacement $A^{-}(\vec{x}) \rightarrow-\mathrm{i} \delta / \delta \chi(\vec{x})$. Then, by making use of the Poisson equation (9.5), we can express $\delta / \delta \chi(\vec{x})$ in terms of $\delta / \delta \alpha(\vec{x})$. Since the functional derivatives will act at the end points, $+\infty$ or $-\infty$, of the Wilson lines we find that

$$
\begin{aligned}
\frac{\delta}{\delta \alpha^{a}(-\infty, \boldsymbol{x})} & =\frac{\delta}{\delta \alpha^{b}(\infty, \boldsymbol{x})}\left[U^{\dagger}(\boldsymbol{x})\right]^{b a} \\
& =[U(\boldsymbol{x})]^{a b} \frac{\delta}{\delta \alpha^{b}(\infty, \boldsymbol{x})},
\end{aligned}
$$

and thus we can express the functional derivatives at $x^{-}=$ $-\infty$ in terms of those at $x^{-}=\infty$. This brings us to the "standard" form of the JIMWLK Hamiltonian

$$
\begin{gathered}
H=\frac{1}{(2 \pi)^{3}} \int \mathrm{d}^{2} \boldsymbol{u} \mathrm{d}^{2} \boldsymbol{v} \mathrm{d}^{2} z K_{\boldsymbol{u}} z \frac{\delta}{\delta \alpha_{\boldsymbol{u}}^{a}(\infty)} \\
{\left[1+U_{\boldsymbol{u}}^{\dagger} U_{\boldsymbol{v}}-U_{\boldsymbol{u}}^{\dagger} U_{z}-U_{z}^{\dagger} U_{\boldsymbol{v}}\right]^{a b} \frac{\delta}{\delta \alpha_{\boldsymbol{v}}^{b}(\infty)},}
\end{gathered}
$$

where we have denoted the dependence on transverse coordinates with an index and with the kernel $K_{u v z}$ given by

$K_{u v z}=\frac{(u-z)}{(u-z)^{2}} \cdot \frac{(v-z)}{(v-z)^{2}}$.

\section{References}

1. E. Iancu (2012). arXiv: 1205.0579

2. Y.V. Kovchegov, E. Levin, Quantum chromodynamics at high energy. (Cambridge University Press, London, 2012)

3. L. Lipatov, Sov. J. Nucl. Phys. 23, 338 (1976)

4. E. Kuraev, L. Lipatov, V.S. Fadin, Sov. Phys. JETP 45, 199 (1977)

5. I. Balitsky, L. Lipatov, Sov. J. Nucl. Phys. 28, 822 (1978)

6. L. Gribov, E. Levin, M. Ryskin, Phys. Rep. 100, 1 (1983)

7. J. Bartels, Nucl. Phys. B 175, 365 (1980)

8. J.L. Albacete, A. Dumitru, C. Marquet, Int. J. Mod. Phys. A 28, 1340010 (2013). arXiv:1302.6433.

9. L.D. McLerran, R. Venugopalan, Phys. Rev. D 49, 2233 (1994a). hep-ph/9309289

10. L.D. McLerran, R. Venugopalan, Phys. Rev. D 49, 3352 (1994b). hep-ph/9311205

11. J. Jalilian-Marian, A. Kovner, A. Leonidov, H. Weigert, Nucl. Phys. B 504, 415 (1997). hep-ph/9701284

12. J. Jalilian-Marian, A. Kovner, A. Leonidov, H. Weigert, Phys. Rev. D 59, 014014 (1998). hep-ph/9706377

13. A. Kovner, J.G. Milhano, H. Weigert, Phys. Rev. D 62, 114005 (2000). hep-ph/0004014

14. E. Iancu, A. Leonidov, L.D. McLerran, Nucl. Phys. A 692, 583 (2001a). hep-ph/0011241

15. E. Ferreiro, E. Iancu, A. Leonidov, L. McLerran, Nucl. Phys. A 703, 489 (2002). hep-ph/0109115

16. E. Iancu, A. Leonidov, L.D. McLerran, Phys. Lett. B 510, 133 (2001b). hep-ph/0102009

17. H. Weigert, Nucl. Phys. A 703, 823 (2002). hep-ph/0004044

18. I. Balitsky, Nucl. Phys. B 463, 99 (1996). hep-ph/9509348

19. Y.V. Kovchegov, Phys. Rev. D 60, 034008 (1999). hep-ph/9901281

20. J.-P. Blaizot, E. Iancu, H. Weigert, Nucl. Phys. A 713, 441 (2003). hep-ph/0206279

21. E. Iancu, D. Triantafyllopoulos, JHEP 1311, 067 (2013). arXiv: 1307.1559

22. K. Rummukainen, H. Weigert, Nucl. Phys. A 739, 183 (2004). hep-ph/0309306

23. A. Dumitru, J. Jalilian-Marian, T. Lappi, B. Schenke, R. Venugopalan, Phys. Lett. B 706, 219 (2011). arXiv:1108.4764

24. Y.V. Kovchegov, J. Kuokkanen, K. Rummukainen, H. Weigert, Nucl. Phys. A 823, 47 (2009). arXiv:0812.3238

25. E. Iancu, D. Triantafyllopoulos, JHEP 1111, 105 (2011). arXiv: 1109.0302

26. E. Iancu, D. Triantafyllopoulos, JHEP 1204, 025 (2012). arXiv: 1112.1104

27. A.H. Mueller, Phys. Lett. B 523, 243 (2001). hep-ph/0110169

28. A. Kovner, M. Lublinsky, Phys. Rev. D 71, 085004 (2005). hep-ph/0501198

29. Y. Hatta, E. Iancu, L. McLerran, A. Stasto, D. Triantafyllopoulos, Nucl. Phys. A 764, 423 (2006). hep-ph/0504182

30. Y. Hatta, Nucl. Phys. A 768, 222 (2006). hep-ph/0511287

31. K. Fukushima, Nucl. Phys. A 775, 69 (2006). hep-ph/0603044

32. F. Gelis, T. Lappi, R. Venugopalan, Phys. Rev. D 78, 054019 (2008). arXiv:0804.2630

33. S. Jeon, Ann. Phys. 340, 119 (2014). arXiv:1308.0263

34. S. Caron-Huot (2013). arXiv:1309.6521

35. I. Balitsky, G.A. Chirilli, Phys. Rev. D 88, 111501 (2013). arXiv:1309.7644 
36. A. Kovner, M. Lublinsky, and Y. Mulian, Phys. Rev. D 89, 061704 (2014). arXiv: 1310.0378

37. D. Binosi, A. Quadri, Phys. Rev. D 84, 065017 (2011), arXiv: 1106.3240

38. D. Binosi, A. Quadri, Phys. Rev. D 85, 085020 (2012a). arXiv: 1201.1807

39. D. Binosi, A. Quadri, Phys. Rev. D 85, 121702 (2012b). arXiv:1203.6637

40. E. Iancu, K. Itakura, L. McLerran, Nucl. Phys. A 708, 327 (2002a). hep-ph/0203137

41. A. Mueller, D. Triantafyllopoulos, Nucl. Phys. B 640, 331 (2002). hep-ph/0205167

42. D. Triantafyllopoulos, Nucl. Phys. B 648, 293 (2003). hep-ph/0209121

43. S. Munier, R.B. Peschanski, Phys. Rev. Lett. 91, 232001 (2003). hep-ph/0309177
44. O. Piguet, S.P. Sorella, Lecture Notes in Physics M 28, 1 (1995)

45. G. Barnich, F. Brandt, M. Henneaux, Phys. Rep. 338, 439 (2000). hep-th/0002245

46. A. Quadri, JHEP 0205, 051 (2002). hep-th/0201122

47. P.A. Grassi, Nucl. Phys. B 462, 524 (1996). hep-th/9505101

48. R. Ferrari, M. Picariello, A. Quadri, Ann. Phys. 294, 165 (2001). hep-th/0012090

49. C. Becchi, R. Collina, Nucl. Phys. B 562, 412 (1999). hep-th/9907092

50. J. Gomis, J. Paris, S. Samuel, Phys. Rep. 259, 1 (1995). hep-th/9412228

51. E. Iancu, A. Leonidov, L. McLerran, (2002b), pp. 73-145. hep-ph/0202270

52. E. Iancu (2014). arXiv:1403.1996 